\title{
Transcending Ethical Dilemmas in Coaching and Supervision: A Model to Develop a Transpersonal Perspective
}

\author{
Karim Hirani \\ London, UK
}

\begin{abstract}
While it is not uncommon to react to ethical dilemmas in coaching with a reactive mindset looking for the "right answer", this approach to dilemmas limits capacity to navigate them as a source of transcendence of conflicts. This paper explores how coaches and supervisors can develop an ethically mature and transpersonal mindset, which allows us to investigate the dilemma in a way that reveals other perspectives and actions that transcend the conflict of the dilemma. Furthermore, the paper offers a six-step process which one can follow when faced with an ethical dilemma, which takes us from developing our mindset through to action and review, with practical examples to demonstrate some of the key steps of the process. While the paper focusses on coaching and supervision examples, the essence of the process can also be used by educators more generally, where they are seeking to transcend conflict and dilemmas in their field.
\end{abstract}

Keywords: ethics, ethical dilemma, transpersonal, ethical maturity, ethical mindset, Kegan, Wilber, Hawkins

\section{Introduction}

"Tell me what to do." This is often the mindset that coaches come and see their supervisor with, seeking out the right answer to an ethical dilemma. Sometimes this mindset can be anxious and reactive, as the worry of getting it wrong or fearing worst case scenarios can overlay a more measured and reflective approach to the problem. This paper is written for coaches and supervisors facing ethical dilemmas in coaching, with the hope that some of the principles of navigating our dilemmas can be applied by the reader to educators and organizations more generally.

Often an ethical issue will activate reptilian brain responses of fear in the face of uncertainty or the unknown (Black and Roberts, 2011). Old patterns of survival, fight or flight may arise. We are put in a new circumstance where a clear response may not be available and many questions arise - do I continue 
the coaching, do I take the matter further with the organization, if I don't do anything will I be in trouble, what is my obligation here, what is expected from my coaching ethical framework, can I break the confidentiality contract, and so forth. We often view the situation as an 'either-or' or 'right-wrong' mindset when we are operating from this frame of reference. We refer to this frame of reference as the ego-identified structure for the purpose of this paper, which, when triggered in the face of a new ethical dilemma, can arouse reptilian brain reactions as well. When we are identified with this triggered ego-identified structure, our options and capacity to see with greater perspective are limited.

The dilemmas we face are often complex and will invariably challenge us to take a deep look at ourselves and our professional practice in order to be in service to the integrity of the profession and our clients.

This paper seeks to explore how we engage in the ethical issues we sometimes face in our coaching from a transpersonal perspective. There are many models already written that focus on ethical decision making processes, such as the work of Rest (1979), Kitchener (1984), Tarvydas (2003) and Carroll (2013). This paper is different in that it focuses more explicitly on:

1. the importance of letting go of identification with our triggered, egoidentified perspective and connecting with a more transpersonal view, that sees the situation from multiple perspectives that are not just personal or ego centred, as is often the case when first faced with the dilemma;

2. how we can transcend the dilemma and find a new resolution;

3. an ethical decision making process that entails a two-fold development of a transpersonal mindset through the processing of the situations we face and which allow transcendent resolutions to emerge.

This paper does not aim to review the literature on ethical decision making processes - the scope of the paper is to introduce and apply a new model pragmatically. However, it is the author's view that contrasting and reviewing against other models would be a valuable endeavor and could form the basis of a future paper.

\section{Some Definitions}

We define an ethical 'issue' as a situation that requires attention of an ethical nature, and an ethical 'dilemma' as an ethical issue where one is faced with apparently conflicting ethics. An ethical issue does not always mean we 
are faced with an ethical dilemma - consider, for example, the situation where we are expected to break confidentiality if we come to know of any terrorist activity through our coaching conversations. There is no dilemma - it must be reported.

We define the ego as the set of mental structures that develops or is patterned primarily through the integration of early experience (Almaas, 1996). A transpersonal view or perspective is a view that goes beyond the ego or the personal self, and we assume here that this view can resolve the apparent opposites of a dilemma (which, as we will see, can 'transcend and include' the different sides of the dilemma, to coin a term from Wilber (2000)). This paper looks at how the view beyond ego can help us with navigating ethical dilemmas we face as a coach.

\section{Two Main Types of Ethical Scenarios}

From the outset, we identify two types of situations when looking at an ethical issue. The first is where there is a clear directive to act. There is no dilemma. These are important to name, as there are situations where there is no deliberation needed. We must check whether our ethical issue comes into this category first.

Otherwise, we see the second scenario - there is no clear directive, and our ethical issue has now become a dilemma. This will become the main focus of this paper, where the need for a transpersonal mindset is helpful in transcending the dilemma. This will be looked at alongside a process that we can use to support this.

\section{Type 1: The Clear-Cut Ethical Scenarios}

Certain ethical issues have clear positions and actions. There is an order of consideration: starting with legal, followed by professional and contractual considerations, which, born out of moral and ethical principles, will often overlap.

Legal

One of the major difficulties here is that the coaching profession is quite new, so there is not much legal precedent that has been set to guide our choices in situations that require legal perspectives (Lindberg, 2006). 
Each country has laws that will vary, one from another. For example, post 9/11, the UK law has positions as follows:

1. Child abuse: one has a responsibility to report child abuse if one becomes aware through one's work of child abuses taking place. One must notify the local child protection team and/or police;

2. Terrorism and money laundering: recent counter-terrorism legislation in the UK is beginning to make it incumbent on professionals to report any knowledge they have of planned terrorist activity or money laundering;

3. Data protection: clients should have knowledge of and access to any data you keep about them electronically. Their details should not be transferred to a third party without their written permission (Hawkins and Smith, 2013).

A further complexity arises when we are coaching clients in different countries.

If we are in any way unclear it is important to seek professional or legal advice when faced with these situations. This is an important perspective to consider when faced with an ethical issue.

\section{Professional}

These include issues that will be addressed through the code of ethics of the accrediting body of the coach (see, for example, http://coachfederation.org/ethicsfaq for the ICF code of ethics).

There are certain clear actions that arise out of such ethical issues e.g. letting a coachee know if there is a conflict of interest (maybe in general terms), stopping coaching if faced with areas beyond your expertise, or saying no to sexual advances from a coachee. Yet even these situations are not always clearcut, which will be discussed later.

These are also issues where you could be called to account for not taking action, though are not required by law to act. For example, reporting 'a person who is a danger to themselves or others.' While there is no legal obligation to act, you could at an inquest be held to account for your response to a coachee if you thought they were a danger to themselves or others (Hawkins and Smith 2013).

Once again, it is important to consider taking professional advice if there is any uncertainty. 


\section{Contractual}

These are ethical issues that are linked to the initial agreement for the coaching relationship, and will also be linked to the code of ethics of the accrediting body of the coach, as the code makes reference to the professional coaching contract.

There are certain clear actions that arise out of such ethical issues e.g. charging for a missed session based on the contractual agreement.

In all of the above clear-cut scenarios, it is advisable to encourage the coachee, where appropriate, to take the right course of action with the understanding that if this does not happen then the coach is obliged to take the matter further out of legal, professional or contractual obligation. It is worth including a statement in the initial coaching contract that is seen by sponsors and coachees.

It is also important that every coach needs to develop their own clear contracting document and ethical principles that are shared with the coachee and client organization at the outset of coaching. These should also include a reference to the professional ethical framework that the coach adheres to and a statement that references one's duty to act when faced with legal or professional issues.

\section{Type 2: Ethical Dilemmas}

The second type of ethical scenarios are not black and white, and we are faced with an ethical dilemma. Many clear-cut scenarios could easily turn into dilemmas. For example, a client has cancelled a session within the 24 hours' cancellation notice but the reason they cancelled is because they had been hospitalised the night before. Do we hold to the pre-agreed contract or do we bring our sense of compassion and waive the cancellation fee? Ethical codes do not address every possible situation, but only provide guidelines and principles upon which we must apply due consideration. As Passmore (2011) writes:

The issue remains, as in nursing, that codes are only a starting point. Codes can never offer a solution for all situations, but only principles to consider in making a decision. The alternative route is to help practitioners develop ethical decision making frameworks which can guide them in making more conscious and informed ethical decisions. (Passmore, 2011) 
Ethical codes can also be limiting. A research study analyzed 27 coaches' ethical challenges, and found three main types of restriction in ethical codes: 1) the code is not relevant; 2) the code has shortcomings; and 3) the code is an obstacle to the ethics of the coach, leading the researchers to conclude that "legitimacy, efficacy, and value issues show that codes do not fit all situations or all practitioners" (Diochon and Nizet, 2015).

This emphasizes the importance of developing a capacity to navigate the dilemmas we face.

The complexity of the dilemma increases further when we consider working across different cultures and the globalization of coaching - we may have a coach in one country coaching a coachee in another country. For example, in the coach's country homosexuality may be legal while it is illegal in the country of the coachee. How does one navigate such issues when one is faced with difference in law, faith and/or culture?

Furthermore, a dilemma for one coach will be perceived by and responded to differently by another coach. Almost every clear-cut scenario is more complex than it appears.

It is to these dilemmas that we now turn our attention for the remainder of the paper.

\section{Navigating Ethical Dilemmas - Transpersonal Mindset and Ethically Mature Attitude}

\section{Development of a Transpersonal Mindset}

The etymology of the word dilemma comes from Greek: di, meaning two, and lemma, meaning proposition, which taken together means a double proposition. An ethical dilemma is a situation where we face more than one competing ethical principle.

For example, recently a coach supervisee attended a supervision session where their coachee had shared with them that they wanted to start a legal suit against their company for unfair treatment. The coach came into the session feeling trapped - on the one hand the coach had been hired by the company to support the coachee with some flexibility on the area to focus on, and on the other hand the coach had professionally contracted to keep the conversation confidential. Clearly triggered, they wanted to know what to do - tell the 
company or keep the information confidential? They were in an ethical dilemma.

As mentioned above, when we are facing the dilemma from our triggered old brain, the tendency is to look at the situation from a place of either-or or right-wrong. We are identified with ego structures, which limit our capacity for creativity and out of the box perspectives.

However, if we develop a capacity to see from a higher perspective in which we can navigate through, respond to and act upon the ethical dilemma, new and transcendent viewpoints can emerge.

Kegan (1994) refers to human development as an evolution in orders of consciousness - each higher order has a greater capacity to embrace complexity and ambiguity to navigate life. Other writers outline similar developments in consciousness, such as Kohlberg's states of moral reasoning (Kohlberg, 1969) and Wilber's stages of human development (Wilber, 2000). Wilber (2000) shows how his model of development aligns with the models of Kohlberg, Kegan and other development theorists' stages.

Taking Wilber (2000) as an example, the development of consciousness moves from ego-centric, world-centric to kosmo-centric. As we transcend the ego-centric world view we are able to see with greater perspective. Furthermore, as we expand our consciousness we are able to access greater compassion and are in line with a higher sense of purpose.

Most importantly, it is through conscious development that we are able to find new and previously unseen perspectives that may not have been available with lower orders of consciousness. As Einstein once said: no problem can be solved from the same level of consciousness that created it. Facing ethical dilemmas requires a shift in consciousness and, furthermore, is sometimes the vehicle through which our own consciousness is raised. As Passmore (2011) writes:

Ethical dilemmas are a route to personal and professional development. We draw on Kelly's (1955) personal construct theory as a way to understand the impact of ethical practice. They suggest that dilemmas are developmental and that they help us to consider what is right and wrong. (Passmore, 2011)

This development of orders of consciousness can give rise to new and potentially transcendent perspectives, as well as other actions that were not 
previously present. In contrast, the triggered reptilian brain has a very simplistic order of consciousness, seeking out a clear right versus wrong answer to the situation.

From a higher order of consciousness, we may also notice that the dilemma is sometimes directly related to the coaching work. For example, a dilemma about whether to charge the coachee for a second missed session, despite the coachee having given good reason, may provide insight into their objective around discipline and commitment. The situation they set up with you may be a parallel for how they may similarly act out in the organization.

To activate our higher order consciousness, we must first be open to developing an ethically mature attitude.

\section{Ethically Mature Attitude}

Developing the right ethical attitude is about noticing the old patterns at play and cultivating an attitude that allows us to mitigate the ethical issue or dilemma through a process of attention, inquiry, action and review that serves the greater good of the coaching. It is about moving through the fear of uncertainty and knowing how to dance with the flow of the emergence of a higher order consciousness and perspective.

Some of the qualities of a transpersonal mindset include curiosity, a willingness to let go of attachment to a perspective, the capacity to be open to new perspectives, to be able to sit with ambiguity, to work through our own barriers, fears and assumptions so that we can navigate the dilemma maturely. Furthermore, through our own self-awareness we may also see how the patterns that have been triggered may provide insight into the dilemma itself. For example, coaching sessions that continually test our ethical boundaries may provide insight into the kinds of dynamics that the coachee sets up.

Furthermore, Caroll (2013) outlines some perspectives on the qualities required for ethical maturity, that include

- Fostering ethical sensitivity and watchfulness - creating ethical antennae that keep us alert to when ethical issues or dilemmas are present;

- Being able to make an ethical decision in the light of ethical principles of our own, our clients, the organisation, society and the profession; 
- Implementing the ethical decision(s) made;

- Being able to articulate and justify to stakeholders the reasons why the ethical decisions were made and implemented;

- Achieving closure on the event and being at peace with it even when there were other possible decisions or better decisions that could have been made.

As mentioned above, we see ethical dilemmas as opportunities for growth and an ethically mature attitude is open to such growth and learning. We are invited and sometimes challenged to move from a lower order of consciousness (where we are also caught by either-ors) to a higher order of consciousness (where we are sitting with the ambiguity, exploring possibilities within the framework of ethical principles, and allowing new courses of action to emerge). We are called to move from seeking 'the' one right answer to exploring multiple perspectives and inviting new and emergent actions. We are challenged to find the courage to act in situations which we may prefer or hope to avoid, offering development and insight for our coachees and their organization. (For example, a Line Manager may wish to divulge information to the coach, that they wish to keep from the coachee. What might this be saying about the coachee-line manager relationship? What might be the culture in the organization that invites triangulation and avoiding direct communication? How might the coach challenge the Line Manager here).

\section{A Six-Step Process for Transcending Ethical Dilemmas}

The six-step process for navigating ethical dilemmas outlined below is informed by the above discussion of mindset and ethical maturity. The process helps us navigate the dilemmas we face, seeking, where possible, to find transcendent perspectives and actions. This process can be used by coaches and supervisors alike, and the principles can apply to educators and organisations too.

It is worth noting that there are two steps in the process that allow for the emergence of higher order perspectives. Step 1 invites this from the outset by cultivating a mature attitude before investigating the dimensions of the dilemma present. Step 3 invites another shift by expanding into a state that invites connection to compassion and our higher purpose in service to the people we work with. 
Here are the steps in the Navigating Ethical Dilemmas process:

\section{Step 1: Cultivating an Ethically Mature Attitude}

In this step we are inviting the coach to notice any triggers, and find a way to resource themselves so that they can stay in an ethically mature mindset. It may mean that in the coaching session where the dilemma is revealed the coach asks the coachee for some time in between sessions before responding to anything that may have triggered the dilemma. Questions to ask include:

What is my state? Reactive or resourceful?

Am I taking this situation from a place of fear or openness and curiosity?

What do I need to resource myself so that I can remain curious and open to learn and serve my client and the situation we face?

What will help me to sit with the ambiguous, the uncertain and the apparently paradoxical?

How can I view this situation from a perspective that allows for emergence of something new?

What are the assumptions I am making right now - about myself, the coach, the situation or the organisation? Which ones are valid and which ones are dramatized/disproportionate?

It may be helpful to bring an attitude or practice of mindfulness before progressing to the next step. This practice can help with relaxing our identification with our patterns and tune into a state of awareness that expands our capacity for greater perspective.

\section{Step 2: Becoming Clear on the Aspects of the Ethical Dilemma}

In this step we are not seeking to find an answer to the dilemma, but are simply being curious and open to what the apparently competing positions we are faced with are. Questions to ask include:

What are the conflicting or variety of ethical positions here, within me and beyond me?

What are the assumptions, frames, and either-ors that are present or could be present in this ethical dilemma? This will include the perspectives that come from legal, contractual, and professional considerations. This will certainly include the code of ethics of our professional body. Different 


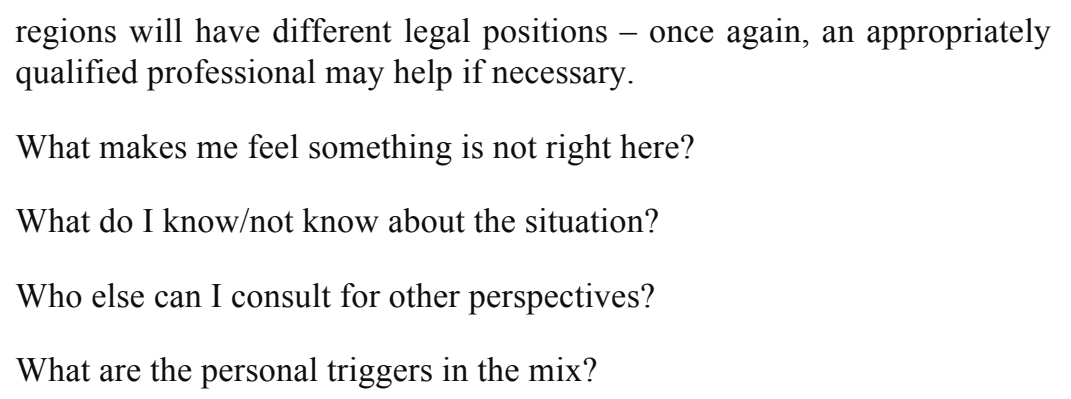

With ethical maturity, we develop the capacity to hold these different positions as we navigate the ethical issue or dilemma.

\section{Step 3: Connect with our Purpose and Compassion}

This step is a second invitation to shift our frame of reference to a higher order of consciousness that invites a deeper ethical maturity.

As Hawkins and Smith (2013) write, "the core of all ethical systems throughout history has been the golden rule that appears and reappears in all the world's major religions. Do unto others what you would like them to do unto you" (p. 250). A more modern version of this rule is the platinum rule, "Do unto others as they would like done unto them." Whichever rule we work with, the point remains the same: the essence of ethical frameworks is born out of a deep care and consideration of the human beings we are working with. It is this quality that we are connecting with here.

Furthermore, Wilber (2000) also affirms that as we develop in our egomaturity, we expand our compassionate embrace, as well as access greater perspective. A connection to purpose also becomes clear, which evokes more choice and invites more possibility.

This step is about raising our order of consciousness, stepping away from the immediate perspectives at hand, and connecting with our highest 'why' that holds compassion for all parts and stakeholders involved.

This may again take the form of doing a short mindfulness practice, stepping back and allowing the space for something new to emerge.

\section{Step 4: Expand to Include Other Frames and Perspectives}

In this step, we are seeking out perspectives that we may not have seen before - opening up our blind spots, challenging our limiting assumptions or 
seeing other possibilities (e.g. systemic and cultural perspectives, other assumptions within and beyond ourselves). This would include viewing the situation from a helicopter view, taking the perspective of being a fly on the wall and sincerely considering the situation with compassion for all parties involved. We could explore the perceptual positions of the different stakeholders.

Questions to ask include:

What are other perspectives that I have not seen before?

What perspectives emerge when we look from different points of view e.g. standing in the shoes of the coachee, organisation, the sponsor, our professional body, from the position of a fly on the wall, an observer or other stakeholders? It is important to genuinely take a moment to step into the worlds of the other with an open heart and mind.

What advice comes from each of these positions?

What is not being said or what am I afraid of saying?

How might other models help me here e.g. how might the model of the drama triangle or transactional analysis inform me about what is happening?

How does the coachee's objective fit into what has been set up in this ethical dilemma? Is something playing out between the coach and coachee that is reflecting a dynamic in the organisation (often referred to as a 'parallel process')?

Through engaging our compassion, higher purpose and perspective in the previous step, we are inviting new perspectives to emerge.

Once more, at this stage we are not seeking to solve the dilemma - we are seeking to allow new perspectives to emerge.

\section{Step 5: Explore Emergent Choices and Take Action}

The purpose of the previous steps is to raise awareness of the range of perspectives we can bring to bear on a given situation, each with their own sense of responsibility. In this step, we are now seeking choices that were not present when first encountering the dilemma. Instead of reacting to the need to find the 'right' answer, we are rising above the dilemma and finding ways to potentially transcend it. 


\section{We are asking ourselves the following questions:}

What are some emergent possible actions?

From these actions, which one feels right?

There are two questions to test the sense of the rightness of the choice and action:

Would we be able to articulate/justify the decision made to all stakeholders?

Does this action feel fair if the tables were turned and we were the coachee (or any other party involved)? ${ }^{1}$

\section{Step 6: Review and Follow Up}

In this final step, we are reviewing the action taken and checking to see if there is resolution around the initial issue or dilemma. It involves exploring the learning, and living with the ambiguity of the moral or ethical decision-making process we have gone through (Caroll, 2013).

\section{Questions to ask include:}

What is the follow up I need to make here to complete this journey? Include the coachee and the organisation in this review.

What have I learned about this situation that I can take forward e.g. updating the terms of my contract?

Are there any other steps above that I need to revisit?

\footnotetext{
${ }^{1}$ It is advisable to avoid triangulation and invite coachees to take the right course of action themselves. This is inviting the coachee to take ownership and responsibility, principles at the heart of coaching. For example, instead of triangulating a legal, professional or contractual issue to HR, it may be a better option to a) invite the coachee to do that and let you know that this has been done; b) challenge the coachee to take action; and/or c) have the conversation with the client about the dilemma you face and the responsibilities you have so that they make a choice on how to act/respond in partnership.

It is also useful to remember that the more we can address and take action with the dilemma at the source point of creation, the simpler the resolution will be. Limiting triangulation and dealing with the situation at source are two good rules of thumb to keep in mind when exploring actions and choices.

We may also have a dilemma that arises out of conflict in roles and relationships with the coachee (e.g. we may be both a line manager and coach - each will have a different guideline on our ethical choice and can be in conflict for ourselves and/or for the coachee). Here, it is important to be transparent and name this up front should any issues arise later on in the coaching relationship.
} 
How can I let go and be complete with this process?

\section{An Applied Example of the Six Step Process for Transcending Ethical Dilemmas}

The step by step process outlined above may be further understood with an example. Instead of focusing on one specific example, however, below is a hypothetical example with multiple possibilities, illustrating some of the many options available to a coach when faced with ethically difficult circumstances.

The example under consideration is of a coach who has been told by their coachee that they have temporarily 'borrowed' a small sum of money from the company expense account, without notifying anyone. This example has been chosen because, while there is only a possible illegal action on the part of the coachee, it shows the other possibilities that can arise if we apply this process, including a transcendent one. The action to report conflicts with our confidentiality agreement, and the law is not clear on our duty to report under these circumstances. Furthermore, in a black and white reaction, the coach may be inclined to react by threatening to take the situation to the organization/authorities, and this reactive mindset may not be the only nor the highest solution available.

In this example, the multiple perspectives or positions on the dilemma (Step 2) might include consideration of:

- Confidentiality between coach and client (code of ethics) versus a professional duty to inform the organization about what the coachee has shared

- Responsibility to the organization that we may have initially contracted with - do we know of or can we find out about company policy

- The position of the law, if any

- Our personal moral position on 'borrowing' money without seeking permission

- Limiting positions or beliefs e.g. avoidance, fear of challenging the coachee or not feeling courageous enough (which may indeed inform us about the power dynamic we have set up with the coachee)

- The contract - is there any reference to this type of situation in the contract 
Step 4 in the process, which involves further expanding frames and perspectives, may involve

- Challenging our limiting assumptions that the coachee has done something 'bad.' What if they were using the money to feed a starving child due to debt? What is their point of view to embrace with compassion?

- $\quad$ Stepping into the shoes of the organization. How might the organization feel about this act? They may be supportive if they understand the situation, and we may challenge the assumption that the result will be a bad one. How might the coachee find out if the organization is supportive or not?

- $\quad$ Systemic perspective. Is this normal practice in the organization and seen as ok? Does the company informally allow this kind of practice?

- What is the coachee's point of view? Have we checked out any assumptions with the coachee in the act of borrowing (e.g. this may be accepted behavior in this organization?)

- $\quad$ Stepping into the shoes of the coachee. The coachee may wish for us to have a direct conversation with them. Have we spoken to the coachee about the dilemma?

- Psychodynamics/models. Noticing the level of responsibility I am taking, what is the responsibility that is not being called out for the coachee or even the organization? Is the coach playing the rescuer, with the coachee being the victim and the organization assumed to be the persecutor? Are we in a parent-child dynamic?

- Models. How might the parent-child dynamic be a parallel or mirror of the same dynamic in the organization or the same dynamic that the coachee is setting up in the organization?

- What is the link to the coachee's objective? For example, the coachee's objective may be linked to their lifestyle or pattern of being in debt.

In this step, we are now seeking choices that were not present when first encountering the dilemma. Instead of reacting to the need to find the 'right' answer, we are rising above the dilemma and finding ways to potentially transcend it.

In this example, Step 5, where new possibilities emerge out of perspectives and frames enlarged in the previous steps, may lead to: 
- Taking the ethical dilemma to the coachee and asking them to report it first. If they agree to do this, then we have transcended our original either-or. We don't need to break confidentiality while upholding our moral or potential legal duty. In this step, we are allowing some new possibilities to emerge once we have opened up the space for different perspectives.

- Explaining your moral position to the coachee. Explain that you will have to act on the situation if the money isn't returned, but that you would like it to resolved by the coachee and explore options with them

- Exploring the issue of responsibility and the coachee's objectives, with a view to having an outcome that will end with the money being returned

- Agreeing to re-contract and set up a three way with the Line Manager for the coachee to explain why they have got to this point in their life, and seek support to act in a way that is aligned with the values and policies of the organization

- If it is informal and normal practice to borrow money in this way, it may be helpful to challenge the coachee to consider how the organization can make this practice formal, open and fair

- If the coachee won't take responsibility to own up, how can you act on your ethical duty with courage and in partnership with the coachee to explain the action you will have to take instead?

Lastly, Step 6, which focuses on review and follow-up, could in this example include:

- Ensuring the coachee has followed through with their actions e.g. returning the money, speaking to the line manager or sponsor

- Ensuring the different stakeholders have been considered in the follow up, as needed

- Updating the coaching contracting to allow a statement that addresses situations where a moral line has been crossed

The possible choices and actions that emerge through this process allow many possibilities that transcend and sometimes include both polarities of the initial dilemma. In this example, the initial polarity of either keeping confidentiality or reporting the issue is no longer present with the suggested choices. We are able to find ways of both holding confidentiality and addressing the issue through our ethical decision making process. 


\section{Another Example of the Six Step Process for Transcending Ethical Dilemmas}

Let's look at another example that demonstrates the steps of the process again. This can be of service to both coach supervisors and coaches reflecting on their own practice.

An organization has contracted with you to coach one of their leaders on an area identified by a recent 360 , supporting the leader to perform better in their role. The coachee says that they wish to leave the company and would like to continue the coaching. The black and white reactive answer may be to discontinue the coaching, as the contract has changed.

\section{Step 1: Cultivating an Ethically Mature Attitude}

Remember to reconnect with the resourceful and ethically mature mindset

\section{Step 2: Become Clear on the Aspects of the Ethical Dilemma}

The sides of the dilemma here are:

- Confidentiality in the coaching (we are not required to break confidentiality here)

- Who is my client - the coachee or the organization? What is the wider purpose of the coaching contract?

- $\quad$ Our contract with the organization to coach the coachee

- $\quad$ Our professional duty as a coach, referring to the code of ethics that we adhere to. For example, the relevant codes from the ICF code of ethics are:

1.4. I will, at all times, strive to recognize personal issues that may impair, conflict or interfere with my coaching performance or my professional coaching relationships. Whenever the facts and circumstances necessitate, I will promptly seek professional assistance and determine the action to be taken, including whether it is appropriate to suspend or terminate my coaching relationship(s).

1.9. I will seek to avoid conflicts of interest and potential conflicts of interest and openly disclose any such conflicts. I will offer to remove myself when such a conflict arises. 
3.15. I will have clear agreements or contracts with my clients and sponsor(s). I will honor all agreements or contracts made in the context of professional coaching relationships.

4.22. I will maintain the strictest levels of confidentiality with all client and sponsor information. I will have a clear agreement or contract before releasing information to another person, unless required by law.

4.23. I will have a clear agreement upon how coaching information will be exchanged among coach, client and sponsor.

\section{Step 3: Connect with our Purpose and Higher Moral Capacity}

Reconnect with our purpose to care for the greater good, including our coachee and organization, beyond the organization and ourselves

\section{Step 4: Expand to Include Other Frames and Perspective}

\section{These may include:}

- Our moral responsibility to both the coach and the organization; our care for our client, organization and beyond

- The responsibility for making the decision is not just ours (it is also the coachee's and the organization's): it is not just our responsibility to seek permission to continue the coaching

- The multi-layered nature of the contract. We had a contract with the coach and with the organization

- The organization has also agreed to pay for the coachee's coaching, so there is an often unsaid contract between the coachee and the organization. Is the coachee honoring this?

- The coach objectives. Is there anything linked here to their objectives e.g. having difficult conversations - limiting beliefs/patterns (pleasing or avoiding)

- What is happening in the organization that is influencing 1) the coachee to leave 2) the coachee to feel that they can't tell anyone;

- Are we assuming that the coachee is not fine with talking to HR about their leaving and asking to continue the coaching?

- Are we assuming that the organization will not be OK with the coaching to continue? 
- What might the organization ask from us?

\section{Step 5: Explore Emergent Choices and Take Action}

Emergent choices may include:

- Being clear with the coach that the contract is now changing so needs to be renegotiated with all parties concerned before coaching can continue

- Requesting that before the coaching continue the coachee addresses the unsaid contract e.g. speak to HR to see if it is OK to continue

- Continuing to work within the current contract if it is true to the original objectives to explore why the coach is not willing to speak to the organization about their impending desire to leave

- Pausing the coaching until the contract has been renegotiated - and agree with the coach around how to honestly communicate this

- If the coach does not agree to have any communication shared, the coach may have to accept termination of coaching (if that is the only option). This will mean that the coach will have to deal with their reactions (e.g. I look like a bad coach if I can't give the real reason why I terminated due to confidentiality). How can the coach get support on this? Or maybe the coach can agree with the coachee to simply name with the stakeholder that there is a conflict of interest that has meant the coaching needs to be terminated (see ICF 1.9 above)

- To bring the dilemma to the next coaching conversation and talk through other possibilities together, while naming the concern around continuing further if the coachee leaves the organization

Finally, take action, ensuring that, if needed, we can justify our choices to all stakeholders involved

\section{Step 6: Review and Follow Up}

What did we learn? Do we need to update our contracting agreement for future coaching engagements? How have we grown? Has the dilemma now been resolved? If not, can we accept the ambiguity? What follow ups are now needed?

Clearly, the perspectives that arise over the course of this six-step process will vary, depending on the coach and/or their supervisor. The intention, however, is that the process offers an expansion in perspective and possibility from the limited perspectives we may start with. 


\section{The Importance of Supervision}

It is the author's view that coaches must make use of supervision when faced with ethical issues and not just with support in working through dilemmas. For example, it may be clear that a coach needs to terminate the coaching if the coachee has shared a mental health disorder and is clearly unable to continue to work professionally with the coachee. The supervision may support the coach in how to manage the communication with the sponsor of the coaching or how to close the coaching with the coachee in a way that leaves the coachee with the right support in place.

Furthermore, supervision is seen to be an important part of one's coaching practice in general. It is through supervision that a coach can be supported to bring their professional integrity to the work that they do and raise their consciousness levels. The process above can be successfully worked through with a supervisor.

For experienced coaches this may take the form of peer supervision. For the novice coach it is recommended that a coach works with a professional supervisor.

\section{Conclusion}

In summary, this paper advocates the importance of expanding our attitude and consciousness when approaching the majority of ethical situations we might face in our coaching. It proposes a model that develops our ethical maturity and transpersonal mindset so that we can potentially transcend ethical dilemmas with clear action and review. It sees the journey as one of consciousness development and growth for all parties involved, in service to the profession, the client(s) and our society.

Through embracing a transpersonal mindset and a process for navigating ethical dilemmas we are finding a way to serve our profession and our clients in the highest possible way. Ethical dilemmas, seen in this way, invite us to grow in consciousness and perspective, and develop resourcefulness, resilience and courage to face these situations that take us to the edge of our comfort zones with the coachees we coach. Rather than be guided by reptilian brain responses and habitual patterns that often make situations worse than they need to be, we are able to invite both the coach and coachee to a place where a higher resolution exists, a journey of expansion that the dilemma can provide. 
With higher order consciousness, we are better able to deal with such issues in an adult partnership and transcend the dilemma in ways that were not available from the outset.

The partnering nature of coaching may be the reason why there is such a low frequency of legal suits and limited legal precedent. The profession itself has the coach-coachee partnership at the heart, so it would make sense that ethical dilemmas can be resolved in partnership without needing to create legal precedents. This is a testament to our profession and a hope for the culture that can be created if we have human relationship at the centre. This process brings us into conscious contact with what is most important when facing our limits in the form of ethical dilemmas.

\section{References}

Almaas, A.H. (1996). The Point of Existence. Boston, MA: Shambhala Publications.

Black, J. \& Roberts, C. (2011). Doing Ethics in Media: Theories and Practical Applications. Oxon, UK: Routledge.

Carroll, M. (2013). Ethical Maturity in the Helping Professions: Making Difficult Life and Work Decisions. London: Jessica Kingsley Publishers.

Diochon, P. F. \& Nizet, J. (2015). Ethical Codes and Executive Coaches: One Size Does Not Fit All. The Journal of Applied Behavioural Science, 51(2), 277-301. https://doi.org/10.1177/0021886315576190

Hawkins, P. \& Smith, N. (2013). Coaching, Mentoring and Organizational Consultancy: Supervision Skills and Development. London: Open University Press.

Kegan, R. (1994). In over our heads: the mental demands of modern life. Cambridge: Harvard University Press.

Kelly. G. (1955). The Psychology of Personal Constructs. London: Routledge.

Kitchener, K.S. (1984). Intuition, critical evaluation and ethical principles: The foundation for ethical decisions in counselling psychology. Counselling Psychologist, 12, 43-55. https://doi.org/10.1177/0011000084123005

Kohlberg, L. (1969). Stage and Sequence: The cognitive-developmental approach to socialization. In D. Goslin (Ed.), Handbook of Socialization theory and research. Chicago: Ran McNally.

Kuhn, T. (2012). The structure of scientific revolutions: 50th anniversary edition. Chicago, IL: University of Chicago Press. http://dx.doi.org/10.7208/chicago/9780226458144.001.0001 
Lindberg, W. \& Desmond, A. (2006). Legal Issues and Solutions for Coaches. In Williams, P. \& Anderson, S. K. (Eds.), Law and Ethics in Coaching. Hoboken, New Jersey: John Wiley and Sons, Inc.

Passmore, J. \& Mortimer, L. (2011). Ethics in coaching. In Hernez-Broome, G. \& Boyce, L. (Eds.), Advanced executive coaching. San Francisco: Wiley.

Rest, J.R. (1979), Development in judging moral issues. Minneapolis: University of Minnesota Press.

Tarvydas, V.M., Cotttone, R.R. \& Claus, R.E. (2003). Ethical decision-making processes. In R.R. Cottone \& V.M. Tarvydas (Eds.), Ethical and professional issues in counselling. Columbus, $\mathrm{OH}$ : Merrill/Prentice Hall.

Wilber, K. (2000). Integral Psychology: Consciousness, Spirit, Psychology, Therapy. Boston, MA: Shambhala Publications, Inc. 